\title{
ON A QUESTION OF N. SALINAS
}

\author{
MUNEO CHŌ
}

\begin{abstract}
In [5], Salinas asked the following question: If $T=\left(T_{1}, \ldots, T_{n}\right)$ consists of commuting hyponormal operators, is it true that (1) $\delta(T-\lambda)=$ $d\left(\lambda, \sigma_{\pi}(T)\right)$ and $(2) r_{\pi}(T)=\left\|D_{T}\right\|$ ? He proved that, for a doubly commuting $n$-tuple of quasinormal operators, (2) was true and (1) was true for $\lambda=0$. In this paper we shall show that (2) holds for a doubly commuting $n$-tuple of hyponormal operators and give an example of a subnormal operator which does not satisfy (1).
\end{abstract}

1. Introduction. Let $H$ be a complex Hilbert space, and all operators on $H$ will be assumed to be linear and bounded. $\mathcal{L}$ will denote the algebra of all operators on $H$.

Let $T=\left(T_{1}, \ldots, T_{n}\right) \in \mathcal{L}^{n}$. We shall say that a point $z=\left(z_{1}, \ldots, z_{n}\right)$ of $\mathbf{C}^{n}$ is in the joint approximate point spectrum $\sigma_{\pi}(T)$ of $T$ if there exists a sequence $\left\{x_{k}\right\}$ of unit vectors in $H$ such that

$$
\left\|\left(T_{i}-z_{i}\right)^{*} x_{k}\right\| \rightarrow 0(k \rightarrow \infty), \quad i=1, \ldots, n .
$$

A point $z=\left(z_{1}, \ldots, z_{n}\right)$ of $\mathbf{C}^{n}$ is said to be in the joint defect spectrum $\sigma_{\delta}(T)$ of $T$ if there exists a sequence $\left\{x_{k}\right\}$ of unit vectors in $H$ such that

$$
\left\|\left(T_{i}-z_{i}\right)^{*} x_{k}\right\| \rightarrow 0(k \rightarrow \infty), i=1, \ldots, n .
$$

The joint numerical range of $T$ is the subset $W(T)$ of $\mathbf{C}^{n}$ such that

$$
W(T)=\left\{\left(\left(T_{1} x, x\right), \ldots,\left(T_{n} x, x\right)\right): x \in H,\|x\|=1\right\} .
$$

The joint operator norm and the joint approximate spectral radius of $T$, denoted by $\left\|D_{T}\right\|$ and $r_{\pi}(T)$, respectively, are defined by

$$
\left\|D_{T}\right\|=\sup \left\{\left(\sum_{i=1}^{n}\left\|T_{i} x\right\|^{2}\right)^{1 / 2}: x \in H,\|x\|=1\right\}
$$

and

$$
r_{\pi}(T)=\sup \left\{\left(\sum_{i=1}^{n}\left|z_{i}\right|^{2}\right)^{1 / 2}:\left(z_{1}, \cdots, z_{n}\right) \in \sigma_{\pi}(T)\right\},
$$

respectively. Let

$$
\delta(T-\lambda)=\inf \left\{\left(\sum_{i=1}^{n}\left\|\left(T_{i}-\lambda_{i}\right) x\right\|^{2}\right)^{1 / 2}: x \in H,\|x\|=1\right\} .
$$

Received by the editors August 10, 1984, and in revised form, February 4, 1985.

1980 Mathematics Subject Classification (1985 Revision). Primary 47A12; Secondary 47B20.

Key words and phrases. Joint approximate point spectrum, joint operator norm, doubly commuting $n$-tuple of hyponormal operators. 
Let $\sigma_{T}(T)$ be Taylor's joint spectrum of $T$. We refer the reader to [7] for the definition of $\sigma_{T}(T)$.

In the sequel, we let $T^{*} T$ be the $n$-tuple $\left(T_{1}^{*} T_{1}, \ldots, T_{n}^{*} T_{n}\right)$.

\section{Theorem.}

THEOREM. Let $T=\left(T_{1}, \ldots, T_{n}\right)$ be a doubly commuting $n$-tuple of hyponormal operators.

Then $\left\|D_{T}\right\|=r_{\pi}(T)$.

We shall need the following three facts.

THEOREM A (CURTO [2]). Let $T=\left(T_{1}, \ldots, T_{n}\right)$ be a doubly commuting $n$ tuple of hyponormal operators.

Then $\sigma_{T}(T)=\sigma_{\delta}(T)$.

THEOREM B (DASH [3]). Let $T=\left(T_{1}, \ldots, T_{n}\right)$ be a doubly commuting $n$-tuple of normal operators.

Then $\overline{W(T)}=\operatorname{co} \sigma_{T}(T)$ (= convex hull of $\left.\sigma_{T}(T)\right)$.

THEOREM C (ChŌ AND TAKAGUCHI [1]). Let $T=\left(T_{1}, \ldots, T_{n}\right)$ be a doubly commuting $n$-tuple of hyponormal operators. If $r=\left(r_{1}, \ldots, r_{n}\right) \in \sigma_{T}\left(T^{*} T\right)$, then there exists a point $z=\left(z_{1}, \ldots, z_{n}\right) \in \sigma_{T}(T)$ such that $z_{i}=\sqrt{r_{i}}(i=1, \ldots, n)$.

PROOF OF THE THEOREM.

$$
\left\|D_{T}\right\|^{2}=\sup \left\{\sum_{i=1}^{n}\left\|T_{i} x\right\|^{2}:\|x\|=1\right\}=\sup \left\{\sum_{i=1}^{n}\left(T_{i}^{*} T_{i} x, x\right):\|x\|=1\right\} .
$$

So there exists $r=\left(r_{1}, \ldots, r_{n}\right)$ in $\overline{W\left(T^{*} T\right)}$ such that $\left\|D_{T}\right\|^{2}=\sum_{i=1}^{n} r_{i}$. By Theorem $\mathrm{B}$, it follows that $\overline{W\left(T^{*} T\right)}=\operatorname{co} \sigma_{T}\left(T^{*} T\right)$. If there exist $\alpha=\left(\alpha_{1}, \ldots, \alpha_{n}\right), \beta=$ $\left(\beta_{1}, \ldots, \beta_{n}\right)$ in $\sigma_{T}\left(T^{*} T\right)$ and $0<t<1$ such that $r=t \alpha+(1-t) \beta$, then we have

$$
\sum_{i=1}^{n} r_{i}<\max \left\{\sum_{i=1}^{n} \alpha_{i}, \sum_{i=1}^{n} \beta_{i}\right\}
$$

which is a contradiction to the choice of $r$. Therefore, $r=\left(r_{1}, \ldots, r_{n}\right)$ belongs to $\sigma_{T}\left(T^{*} T\right)$ and there exists $z=\left(z_{1}, \ldots, z_{n}\right)$ in $\sigma_{\delta}(T)$ such that $\sum_{i=1}^{n} r_{i}=|z|^{2}$ by Theorems $\mathrm{C}$ and $\mathrm{A}$.

Let $\left\{x_{k}\right\}$ be a sequence of unit vectors in $H$ such that

$$
\left\|\left(T_{i}-z_{i}\right)^{*} x_{k}\right\| \rightarrow 0(k \rightarrow \infty), \quad i=1, \ldots, n .
$$

Hence,

$$
\sum_{i=1}^{n}\left\|\left(T_{i}-z_{i}\right) x_{k}\right\|^{2} \leq|z|^{2}-2 \cdot \sum_{i=1}^{n} \operatorname{Re} \bar{z}_{i}\left(T_{i} x_{k}, x_{k}\right)+|z|^{2},
$$

and, since $\left(T_{i} x_{k}, x_{k}\right)=\left(x_{k}, T_{i}^{*} x_{k}\right)=\left(x_{k},\left(T_{i}-z_{i}\right)^{*} x_{k}\right)+z_{i} \rightarrow z_{i}(k \rightarrow \infty), i=$ $1, \ldots, n$, we have

$$
\sum_{i=1}^{n}\left\|\left(T_{i}-z_{i}\right) x_{k}\right\|^{2} \rightarrow 0, \quad \text { as } k \rightarrow \infty
$$

Therefore, $z=\left(z_{1}, \ldots, z_{n}\right)$ belongs to $\sigma_{\pi}(T)$.

So the proof is complete. 
3. Examples. Let $H$ be an infinite-dimensional Hilbert space with orthonormal basis $\left\{e_{k}\right\}_{k=1}^{\infty}$. Consider the weighted shift $T$ on $H$ such that

$$
T e_{1}=\frac{1}{2} \cdot e_{2}, \quad T e_{k}=e_{k+1} \quad(k=2,3, \ldots) .
$$

Then $T$ is subnormal (cf. p. 379 in Stampfli [6]), $\sigma_{\pi}(T)=\{z:|z|=1\}$ (cf. Furuta [4]) and evidently $\delta(T)=\frac{1}{2}$. Therefore, $\delta(T)<1=d\left(0, \sigma_{\pi}(T)\right)$, so that (1) is in general false for a subnormal $n$-tuple.

ACKNOWLEDGMENT. We would like to express our cordial thanks to the referee and Professor Furuta for their kind advice.

\section{REFERENCES}

1. M. Chō and M. Takaguchi, Some classes of commuting n-tuple of operators, Studia Math. 80 (1984), 245-259.

2. R. E. Curto, Fredholm and invertible $n$-tuples of operators, The deformation problem, Trans. Amer. Math. Soc. 266 (1981), 129-159.

3. A. T. Dash, Joint numerical range, Glasnik Mat. 7 (1972), 75-81.

4. T. Furuta, A counterexample to a result on approximate point spectra of polar factors of hyponormal operators, Yokohama Math. J. 32 (1984), 224-225.

5. N. Salinas, Quasitriangular extensions of $C^{*}$-algebras and problems on joint quasitriangularity of operators, J. Operator Theory 10 (1983), 167-205.

6. J. G Stampfli, Which weighted shifts are subnormal?, Pacific J. Math. 17 (1966), 367-379.

7. J. L. Taylor, A joint spectrum for several commuting operators, J. Funct. Anal. 6 (1970), 172-191.

Department of Mathematics, Joetsu University of Education, Joetsu 943, JAPAN 